Recherches en didactique des langues et des cultures

Les cahiers de l'Acedle

17-2 | 2020

Recherches collaboratives en didactique des langues

\title{
Les jeux de la recherche collaborative : le cas du projet artistique
}

Maud Serusclat-Natale et Yannick Marzin

\section{OpenEdition}

Journals

Édition électronique

URL : https://journals.openedition.org/rdlc/7712

DOI : $10.4000 /$ rdlc. 7712

ISSN : $1958-5772$

Éditeur

ACEDLE

Référence électronique

Maud Serusclat-Natale et Yannick Marzin, « Les jeux de la recherche collaborative : le cas du projet artistique ", Recherches en didactique des langues et des cultures [En ligne], 17-2 | 2020, mis en ligne le 27 avril 2020, consulté le 17 octobre 2022. URL : http://journals.openedition.org/rdlc/7712 ; DOI : https://doi.org/10.4000/rdlc.7712

Ce document a été généré automatiquement le 17 octobre 2022.

\section{c) (i)}

Creative Commons - Attribution - Pas d'Utilisation Commerciale - Pas de Modification 4.0 International - CC BY-NC-ND 4.0

https://creativecommons.org/licenses/by-nc-nd/4.0/ 


\title{
Les jeux de la recherche collaborative : le cas du projet artistique
}

\author{
Maud Serusclat-Natale et Yannick Marzin
}

1 Cet article retrace à deux voix (distinguées typographiquement) une expérience de collaboration en cours entre une doctorante en sciences du langage et un directeur de scène nationale travaillant ensemble autour d'un projet nommé Parlemonde, à la fois programme de création artistique pluriel et plurilingue, et objet de recherche. Yannick Marzin (désormais YM, dont l'écriture est visible en italique dans le texte) apporte son regard professionnel sur le projet, en complémentarité avec celui de Maud SérusclatNatale, doctorante et intervenante ${ }^{3}$. Nous traiterons particulièrement de la question du statut du chercheur embarqué dans et par les dynamiques de création artistique. Nous arrivons au terme d'une année de collaboration mais notre relation repose sur des années de travail en commun sur un même territoire. Nous signons ensemble ce qui correspond à l'état actuel de nos discussions alors que s'est achevée la collecte des données de recherche, et que commence une seconde année dédiée à leur exploration et à la préparation du prochain Parlemonde.

2 Nous examinerons d'abord la construction de cette collaboration, puis nous nous interrogerons sur les espaces de jeu, au sens d'intervalles entre deux pièces d'un même mécanisme, que suscite la collaboration, en nous intéressant particulièrement aux conséquences sur les données de recherche. Enfin nous évoquerons ses enjeux dans ce contexte agissant au cœur de l'espace social.

\section{Du partenariat à la collaboration : quelles règles du jeu?}

3 Parlemonde ${ }^{4}$ est organisé par une scène nationale publique ${ }^{5}$ à Montbéliard (France) depuis 2017 en partenariat avec l'Éducation nationale. Ce festival met à l'honneur les créations participatives réalisées à partir des langues et des cultures de chacun. Il met 
en scène la rencontre au sens large: rencontre avec soi, avec son nouvel espace de vie, les autres, les publics et les arts vivants. Les collaborations école/culture sont habituelles et les politiques nationales ou locales les encouragent vivement, en vue de favoriser l'engagement, la motivation ou l'estime de soi des élèves, mais également pour apaiser le climat scolaire ou participer à la cohésion sociale, pour reprendre les termes de l'éducation nationale. École et Institution culturelle publiques partagent une mission commune essentielle : l'éducation artistique et culturelle. Plusieurs dispositifs existent pour cela, au-delà des disciplines artistiques inscrites dans les curricula, notamment depuis la création du parcours d'éducation artistique et culturelle (PEAC) ${ }^{6}$ en 2013, que chaque élève alimente de son entrée à l'école à la fin de sa scolarité.

\section{Institutionnaliser la collaboration entre le chercheur et les praticiens}

4 L'expérience Parlemonde 1 s'est déroulée sur l'année scolaire 2016/2017. Il s'agit pour les participants et $\mathrm{MA}^{7}$ d'une première expérience à cette échelle, dans le sens où elle a fait travailler ensemble de nombreux acteurs : 5 artistes sont engagés auprès de 4 établissements scolaires et d'un centre d'hébergement pour mineurs non accompagnés soit une dizaine d'enseignants, 80 élèves et les équipes artistiques et techniques. 6 créations sont nées de ces collaborations et ont été présentées sur scène en mai 2017.

5 A l'issue de 8 mois de résidences et de 2 jours de festival, les artistes et les élèves témoignent de leur envie de poursuivre, tout comme les partenaires éducatifs. Depuis, Parlemonde est devenu un festival déployé en biennale intégrant tous les deux ans la programmation de MA et instaurant une collaboration avec l'éducation nationale et les EANA au long cours.

6 La collaboration de recherche s'est trouvé scellée un an après Parlemonde 1 à travers deux types de conventions : la première, dans une convention industrielle de formation par la recherche (Cifre) ${ }^{8}$ portée par MA avec l'université Paul Valéry de Montpellier. La seconde a été signée avec le rectorat de l'académie de Besançon, permettant à la doctorante de poursuivre les échanges avec les équipes pédagogiques et les formateurs du CASNAV et de la DAAC, car l'un des enjeux de Parlemonde est de venir nourrir des changements de paradigme didactique.

\section{Le projet Parlemonde : créer en contexte multilingue}

7 Le terrain de la collaboration culture/école intéresse depuis longtemps des chercheurs comme Dupont (2010), Bordeaux (2014, 2017) ou Kerlan \& Lemonchois (2017), dont les travaux ont montré à la fois le grand intérêt de ce type d'expérience pour les élèves, mais également les tensions que les partenaires peuvent rencontrer en travaillant ensemble, ainsi que les difficultés à en évaluer les effets dans un temps compatible avec les enjeux de la recherche (Bamford, 2008).

Parlemonde a déplacé considérablement le cadre habituel des collaborations entre les deux institutions. En effet, il s'agit de co-construire avec des acteurs n'ayant jamais travaillé ensemble (artistes, élèves allophones nouvellement arrivés - désormais EANA-, enseignants) une démarche de création et de production d'œuvres artistiques plurielles et plurilingues à l'école, en mobilisant particulièrement les EANA du secondaire sur le temps scolaire dans le cadre de résidences artistiques. Les interventions s'étendent sur un temps long (entre 60 et 100 heures d'intervention en 
classe) et proposent parfois des périodes de travail intense ( 2 ou 3 journées d'affilée ou une semaine, puis des intervalles réguliers). Or le temps scolaire est extrêmement contraint et les moments de résidence bousculent l'organisation des enseignements disciplinaires, à la différence d'un cours de «théâtre » ou d'un atelier spécifique qui aurait lieu chaque semaine.

Dans les « fiches action » élaborées par les enseignants porteurs de projet à l'institution scolaire, le travail avec les artistes doit servir de support au «développement des compétences linguistiques », « favoriser l'inclusion des EANA » dans l'établissement et "dé-ségréguer » les dispositifs UPE2A. Leur participation à Parlemonde vise donc à valoriser les compétences plurilingues des EANA tout en accélérant leur appropriation du français. Cet objectif veut favoriser l'inclusion des élèves par un accompagnement répondant à leurs besoins particuliers 9 (Auger 2001; Pierra 2006 ; Auger et al. 2007 ; Auger et al. 2009 ; Sérusclat-Natale \& Adam-Maillet 2018).

Pour MA scène nationale, Parlemonde dépasse la collaboration habituelle avec l'École parce que ce festival intègre sa programmation artistique. Ses résidences s'inscrivent dans son projet artistique global, qui pour YM mise sur son implantation locale et, dans un grand écart voulu et assumé, ouvre des fenêtres sur le monde. Parlemonde répond de la même exigence artistique que les spectacles produits et joués par les compagnies professionnelles : il y a une "direction artistique», une signature dans le concept même de l'événement avec des orientations qui démarrent par le choix des artistes, de leur discipline et ensuite le choix du lieu. C'est cet aspect qui fait la différence entre des ateliers et des productions qui s'inscrivent dans un événement construit. Né en 2017 mais préparé dès 2015, Parlemonde s'ancre dans un contexte médiatique marqué par « la crise migratoire », les crispations identitaires multiples et les nombreux appels au monde de la culture pour favoriser «l'intégration» des "migrants" ${ }^{10}$. Afin de favoriser les rencontres, les créations Parlemonde sont mises en valeur publiquement lors d'un festival de plusieurs jours au cœur de la ville. Les enjeux et les prises de risques sont donc importants tant pour les artistes que pour les écoles.

11 Si les deux partenaires sont prêts à travailler ensemble et se connaissent de longue date, ils ne parlent pas vraiment la même langue car ils relèvent de métiers, cultures et représentations différentes. De plus, les 250 participants plurilingues de Parlemonde font circuler une trentaine de langues (sans compter les langages de la création artistique), de multiples langues professionnelles des acteurs impliqués ainsi que leurs habitus et cultures disciplinaires : les questions d'explicitation des univers de référence de l'Autre (artiste, enseignant, élève, assistant, chargé de mission, doctorant etc.) n'ont eu de cesse de se poser. Notre choix de faire apparaître nos deux voix distinctes dans cet article souligne cet élément réflexif central: le 4 mains c'est aussi au moins deux champs avec des codes et des langages qui doivent être explicités pour s'accorder, non?

\section{Définir la collaboration}

12 Collaborer, c'est dépasser le paradigme du "partenariat " (un partenaire utilisant les compétences de l'autre et réciproquement, chacun dans un but inhérent à son domaine) pour que s'installe un "processus de recherche et de transformation des pratiques » (Vinatier \& Morrissette 2015 : 159) dans lequel « l'implication des praticiens est mobilisée » (Bednarz 2015 : 173). Par sa nature artistique et multimodale, l'objet et le projet Parlemonde ont constamment évolué. Ce caractère dynamique l'inscrit dans le 
paradigme de la collaboration : il ne s'agit pas seulement de travailler ensemble mais, chemin faisant, d'être transformé par la collaboration et de faire évoluer ses pratiques dans son champ professionnel. Cette expérience peut être représentée ainsi :

Figure 1- L'expérience de collaboration Parlemonde

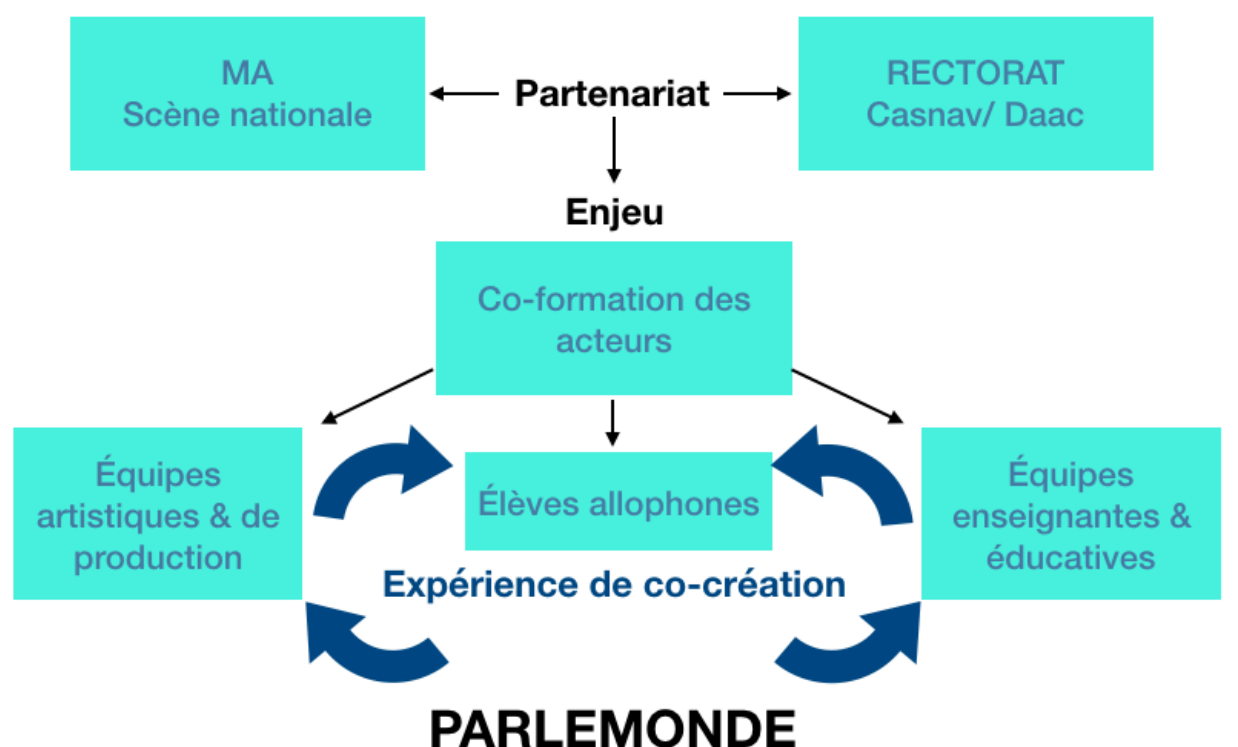

L'expertise de chacun est requise, de façon dynamique, pour qu'une œuvre commune naisse. Pour chacun, cette expertise évolue au contact des autres participants. Ici l'implication des praticiens est mobilisée à chaque étape et trois niveaux de collaboration sont requis pour créer l'œuvre qui sera présentée publiquement: une collaboration entre responsables institutionnels (CASNAV, DAAC et structure culturelle), une collaboration entre équipes artistiques et équipes enseignantes en classe, une collaboration entre élèves et artistes. S'y mêle dans le même temps une collaboration entre l'ensemble des acteurs du projet (professionnels, artistes, enseignants, élèves) et la doctorante, qui s'intéresse à la fois à situer l'impact des expériences artistiques dans l'appropriation d'une langue cible pour les EANA, et dans la construction d'un geste professionnel inclusif pour les enseignants et les équipes artistiques.

Pour YM, la particularité de Parlemonde a été d'évoluer avec l'impact et la force du projet. C'est parce que tu as décidé d'en faire ta thèse et que nous nous sommes mis d'accord pour te faire intégrer l'équipe et que nous avons commencé à penser collectivement, et c'est ainsi que le projet a évolué pour devenir ce que nous en faisons aujourd'hui, du festival à un programme avec des résidences, des séminaires, des publications et des productions Parlemonde, comme la prochaine pièce de Charlotte Lagrange, L'Araignée.

La collaboration n'est pas une superposition ou une succession d'actions commises par différents acteurs en jeu mais une action partagée et ses évolutions posent de nombreuses questions, tant techniques (comment ne pas figer la collaboration?) que méthodologiques et déontologiques, dans la mesure où, comme le rappellent Blondiaux, Fourniau \& Mabi « "travailler avec » n'a pas tout à fait le même sens que «travailler pour » » $(2016: 10)$. Elle relève d'une dynamique. L'enjeu de la recherche est 
d'éclairer cette dynamique : comment naissent et circulent les éléments permettant l'agir ensemble constitutif d'une activité de création artistique? En quoi le plurilinguisme soutient-il cette action? Plus particulièrement: quels sont les effets d'une collaboration entre chercheur et praticiens dans ce contexte?

\section{Un « embarquement » réciproque : conséquences épistémologiques}

\section{Du poste à la posture du « chercheur embarqué » ou « embarquant »}

La position de salarié du doctorant Cifre constitue un "cadre de recherche singulier " (Ferrando y Puig \& Petit, 2016: 20) largement documenté sous la forme du paradoxe par les chercheurs (Hellec, 2014), comme l'évoquent Vinatier \& Morrissette:

«Ce dispositif [...] place le doctorant, jeune salarié dans l'organisation, dans des positions complexes de tenue de multiples rôles, reliés à la recherche scientifique dans son laboratoire et à l'opérationnalité de ses analyses dans l'organisation recruteuse. Cette hybridation de la recherche et des enjeux économiques de l'entreprise risque d'obliger restrictivement le doctorant à répondre aux commandes institutionnelles avec une stricte posture de recherche appliquée » (2015: 157).

17 La position de « doctorante associée ${ }^{11}$ » à une scène nationale, observant, participant et co-construisant le projet qui constitue également son objet de recherche est une « expérience incorporée » (Ferrando y Puig \& Petit, 2016: 20) inédite. Dans une telle structure de création, « embarquée » par un tel projet artistique, définir un poste précis s'est avéré difficile car on attendait plutôt que la doctorante occupe plusieurs rôles transversalement. Blondiaux, Fourniau \& Mabi rappellent que c'est souvent le cas lors des recherches collaboratives : «il est impossible [...] de résumer le rôle du chercheur dans les processus participatifs à une posture unique» (2016: 10). Pour YM, il faut montrer à l'équipe comment cette création de poste participe au projet général de la maison et accompagner une modification des équilibres et des habitudes de fonctionnement. Lorsque la création d'un tel poste se réfere à un statut inhabituel pour une entreprise culturelle, cela demande de faire preuve d'une plus grande pédagogie vis à vis de l'équipe et des tutelles qui valident la création d'emploi lors des Conseils d'Administration de l'association. La Cifre a donc conduit à de nombreuses réorientations du travail de recherche: «l'activité professionnelle génère et oriente l'activité de recherche [qui à son tour] ressource et réoriente l'activité professionnelle» (De Lavergne, $2007: 29$ ). Cela a des conséquences sur les méthodologies, les données et les finalités que chacun (entreprise, directeur de recherche et doctorante) se représente de la thèse en cours, qui, d'une certaine manière, échappe parfois au chercheur puisqu'elle devient, en chemin, commanditée. Le partenaire professionnel attend notamment qu'elle présente des effets opératoires, c'est-à-dire qu'elle génère des changements de gestes professionnels appréciables. Pour YM, l'objectif est d'utiliser et jouer avec l'impact des recherches appliquées pour faire évoluer le projet général de la maison si jamaisj'y voyais le potentiel. Le statut de doctorante associée fut le résultat de la première validation de l'intérêt à court et moyen termes (3 à 5 ans) pour la scène nationale dans le cadre de ses missions "éducatives ». [...] J'attends des connaissances de M. et de la dimension "recherche" de son travail, un élan à partir duquel je pourrais créer une dynamique de changement et de questionnement interne pour revitaliser la réflexion et ne pas limiter nos métiers à de la médiation ou de la production dans un rapport binaire avec les 
publics comme avec les artistes. Une doctorante en action était aussi un motif de réintroduction de la pensée, auprès d'une équipe dont la majorité des membres est titulaire d'un Master.

On peut donc parler d'un embarquement réciproque dans une collaboration dont les enjeux ont évolué. Les intérêts didactiques et éthiques de Parlemonde ont conduit la formatrice CASNAV à devenir doctorante et le directeur de la scène nationale à créer un poste de recherche -développement transversal nourrissant sa propre réflexion à la tête de l'entreprise, son équipe et les artistes.

\section{De la posture à la relation}

Les pratiques artistiques exposent et s'exposent, ce qui crée du lien. La collaboration relève de la même dynamique. La création artistique en collaboration revêt un enjeu à la fois social, éducatif, linguistique qui, au fil de l'expérience, a rendu visible le besoin d'interaction et de médiation. Cela a ancré le travail du chercheur dans une épistémologie de la relation puisqu'en effectuant des entretiens, il se tient entre mais surtout parmi, avec, au cœur de son terrain et de l'acte même de créer ${ }^{12}$.

Les artistes ont été les premiers à investir la doctorante en tant qu'interlocutrice « initiée » puisque salariée par MA, ou en tant qu'interface de médiation faisant le lien entre les élèves, les enseignants et le milieu de la création artistique. YM : En effet tu te trouves dans une légitimité que n'ont pas les autres membres de l'équipe. De mon point de vue tu te situes à un endroit semblable à celui que les artistes connaissent quand ils démarrent un processus de création/recherche. Tu représentes un apport réflexif qui anime leurs recherches et peut orienter leur création. Il y a un espace de création au sens fort du terme auquel tu appartiens. La question de la légitimité est extrêmement sensible et subjective dès lors que tu es là avec eux, in situ. Les entretiens avec les artistes ont été très faciles à recueillir (malgré les contraintes géographiques et linguistiques diverses) et les dialogues se poursuivent encore, au-delà de la collecte de données désormais close. Ils ont investi la fonction du chercheur, mais de façon très différente, comme on le voit dans les extraits ci-dessous. Ces extraits de corpus émanent de 2 artistes ayant participé aux 2 Parlemonde, collaborant avec la doctorante depuis 3 ans, de manière ponctuelle mais régulière.

Extrait $1^{13}$ :

$\mathrm{D}$ : Comment as-tu vécu ou vis-tu notre collaboration?

ENTA1 : Notre collaboration a modifié beaucoup de choses pour moi, petit à petit. $\mathrm{Tu}$ étais dans un accompagnement jamais pédagogique et pourtant hyper instructif. Tu m'as laissée me faire mon opinion tout en exposant ton regard par petites pincées de sel...Et ces pincées m'ont guidée dès le début du travail pour Sédiments et dans les réflexions politiques et dramaturgiques qui ont suivi et qui sont à l'origine de L'Araignée... C'est aussi l'humain, c'est une rencontre... ça passe par là, et par le temps aussi, on a pris le temps de cette recherche en commun.

Extrait 2:

D : Maintenant qu'on se connaît depuis 3 ans, comment tu décrirais ce que je fais moi dans tout ça?

ENTA2 : [19 secondes d'hésitation] Au début en fait, d'un côté c'est comme des... ce n'est pas vraiment comme quelqu'un des NGO's...Mais...Tu es comme une personne qui prend aussi soin de ces jeunes, qui est attentive pour qu'ils aient une possibilité d'autonomie ici et... aussi ailleurs en fait, et après tu es passionnée par les langues. Je me rappelle pour Les Portes quand on a parlé avec les élèves et cherché dans leurs langues des mots, des textes et des proverbes, tu as amené ça! Pour moi c'est la passion qui te définit. Tu es 
passionnée. Après l'élément du doctorat finalement je crois que ce n'est pas important, c'est la passion, la patience et la recherche aussi, tu cherches un chemin...Comme le font les artistes, eux aussi sont souvent passionnés, fascinés par quelques chose et toi, c'est ça, avec les langues des gens.

21 C'est comme cela que MA fonctionne, on va chercher des collaborations AVEC des artistes pour qu'ils m'accompagnent dans mon projet dans une relation réciproque. Je les accompagne à mon tour de façon différenciée. Ta pratique à mes côtés t'a fait passer par cela. Ta recherche répond à un schéma semblable et ton lien avec les artistes ou des artistes avec Parlemonde suit le même scénario. Il me semble important de personnaliser car il y a un va et vient constant entre les personnes et les maisons ou institutions. Mais le départ naît d'une volonté et d'un choix personnel et subjectif qui passe par l'établissement d'un lien qui est nourrit et se développe en complicité permettant un aller retour constant aboutissant à la production finale. L'embarquement devient un principe empirique et subjectif de fonctionnement entre les artistes et moi, entre toi et moi, les artistes et toi.

Ce lien dépasse la relation directeur- artistes- doctorante associée. Les élèves, notamment les lycéens, ont investi la posture d'écoute et de médiation du chercheur, certains demandant parfois à interrompre le travail avec l'artiste pour lui « parler » ou «s'enregistrer » avec elle, considérant qu'elle était « celle qui gardera une trace de ce que nous avons à dire sur ce que nous faisons ${ }^{14} »$.

Ce lien particulier entre toi et les jeunes a influé sur toute la démarche de création. Je te sens très proche des jeunes à un endroit non touché par les autres intervenants et adultes d'une manière générale. Pourquoi? Ta complicité avec eux permet autant qu'accompagne et se nourrit du lien tissé avec le créateur.

Cette présence ethnographique a bien entendu été remarquée par les enseignants qui, constatant ce lien avec les élèves, se sont livrés avec plus d'aisance lors des entretiens de fin de projet, et ont expliqué qu'ils «n'avaient plus de crainte » particulière ou qu'ils pouvaient « se lâcher » plus facilement. Si ces liens ont grandement aidé à approfondir le questionnement de recherche, ils posent des questions éthiques et épistémologiques importantes.

\section{Être à la fois (du) dedans et (du) dehors}

\section{Assumer un rapport subjectif au(x) terrain(s)}

S'il n'y a pas de chercheur qualitatif objectif (Pourtois \& Desmet, 2009 : 304) et si une recherche ethnographique n'est pas modélisable ou généralisable hors contexte, il faut assumer que son terrain n'est pas un terrain de recherche neutre mais plutôt le terreau de la chercheure en devenir ${ }^{15}$, terreau multilingue et pluriel : " [Un terrain] c'est d'abord un ensemble de relations personnelles où " on apprend des choses » et " faire du terrain ", c'est établir des relations personnelles avec les gens » (Agier, 2004 : 35). En rendre compte en tant que chercheure-doctorante embarquée, c'est reconnaitre les «jeux» dans la relation, et assumer le «je» dans l'écriture. Dans mon cas, cette relation s'inscrit dans une histoire vécue préexistant la collaboration: j'ai grandi à Montbéliard, j'y ai enseigné puis j'y ai formé des enseignants. Parallèlement, j'ai animé des ateliers théâtre en partenariat avec MA, collaboré à des projets participatifs (parfois en langue étrangère) impliquant la jeunesse sur mon temps de travail d'enseignante et en dehors. Les acteurs des deux terrains, scolaire et culturel, me 
connaissent de longue date, tant personnellement que professionnellement et ces liens ont fait naître Parlemonde. Ma position de chercheure "embarquée " (Alam, Gurruchaga \& O'Miel 2012) a favorisé l'émergence de dialogues que je n'aurais pu prévoir avec les participants. Cette position constitue «bien souvent une ressource, beaucoup plus qu'un obstacle " (Ferrando y Puig \& Petit $2016: 10$ ). Les frontières entre recherche et action, ou participation à la vie du terrain, sont poreuses : ce terrain d'expérience est ici un terrain partagé.

\section{Effets sur le corpus}

Jouer la collaboration ouvre un espace interstitiel qui intéresse conjointement le doctorant et le commanditaire, un espace de dialogue à la fois formel et informel inédit de part et d'autre, instauré et instaurant des pratiques de réflexivité. Ce jeu repose sur un équilibre toujours à construire entre utilisation d'une expertise acquise par l'expérience de terrain et élément catalyseur ou moteur de réflexivité inhérente à l'activité de recherche. Sur ce terrain, les interactions sont extrêmement riches et variées et forment un corpus très important qui a été recueilli durant Parlemonde 1 et 2 de façon multimodale par le biais d'enregistrements audio, vidéo, de notes de terrains, de questionnaires et d'entretiens semi-directifs. Mon protocole s'intéressait dans un premier temps davantage aux interactions élèves/artistes/enseignants collaborant lors des résidences et s'est trouvé suite à la Cifre, complété par des entretiens avec les artistes et les membres des équipes de production, de communication et de relations publiques de la structure, éléments dont l'analyse intéresse davantage l'entreprise qui me considère comme un "agent de mouvement". Par interaction, j'entends donc « relation », rejoignant Blanchet : " au sens complet de la relation humaine et sociale [...] une interaction engage toujours la totalité des personnes et des contextes (leurs histoires, leurs représentations, leurs tensions, leurs altérités, etc.) » $(2012: 124)$

\section{Les conflits de temporalités}

Parlemonde a fait travailler ensemble des univers singuliers, culturellement et linguistiquement marqués, qu'il s'agisse de mobiliser les élèves nouvellement arrivés et non encore locuteurs du français pour certains, les équipes enseignantes non familières avec la création artistique, ou les artistes non familiers avec les créations participatives plurilingues en milieu scolaire. Comme toute expérience et rencontre avec ce qui est "autre", "nouveau», échappant à des rituels confortables, l'expérience de la collaboration dans un projet artistique met à l'épreuve, bouscule car il y a interculturalité comme le rappelle Auger: "La complexité culturelle de chacun, traversée d'éléments collectifs et singuliers, fait de chaque rencontre une rencontre interculturelle» (2007: 13). Dans ces circonstances, tout à fait propices et même nécessaires à la création artistique, je suis devenue à la fois celle qui cherche, observe, observe-participe, et intervient, en donnant un éclairage sur une situation précise, sans attendre. Je partageais donc le jeu et instaurais des temps de réflexivité qui confinaient à de l'analyse de la pratique presque immédiate, afin d'éviter des écueils qui auraient pu nuire non pas seulement à l'œuvre artistique en train de naître, mais avant tout au bien-être des participants. 

initiative ou sur les sollicitations des acteurs (élèves, équipes, artistes). Tant que l'expert est dans son équipe et dans l'action du terrain, cette réactivité présente une rentabilité immédiate pour la structure. Mais comment dépasser le statut d'expert associé à une démarche ou à un projet? Cette posture se rapproche du "marginal sécant » explicitée par Ferrando y Puig \& Petit en ces termes :

«Partie prenant dans plusieurs systèmes d'action en relation les uns avec les autres, et qui peut, de ce fait jouer le rôle indispensable d'intermédiaire et d'interprète entre des logiques d'action différentes, voire contradictoire. [...] Cette division du travail réflexif, si elle peut permettre une progression mutuelle, risque d'aboutir à une perspective utilitariste faisant de cette réflexivité une caution de scientificité sans réelle portée critique » $(2016: 28)$.

Cette question n'est pas encore tranchée en ce qui me concerne. Trois problèmes majeurs sont à souligner quant au positionnement scientifique dans une collaboration de ce type. Tout d'abord la légitimité de la caution que la présence du chercheur apporte n'est pas tant liée à son expertise scientifique (au sens où elle serait liée au statut de doctorant ayant des connaissances théoriques dans un champ donné ici la didactique des langues), qu'à son expérience professionnelle et interculturelle antérieure. Ensuite les rétroactions qui sont conduites dans une temporalité immédiate laissent peu de place à une analyse approfondie des phénomènes observés, distanciation scientifique pourtant nécessaire. Se pose en dernier lieu la question de la transmission de mes compétences de médiation et d'analyse aux équipes.

La principale limite reste alors, comme le soulignent Vinatier \& Morrissette dans leur état des lieux des recherches collaboratives, la temporalité: "parce qu'elle[s] s'inscri[ven]t dans la longue durée, [les recherches collaboratives] déroge[nt] aux normes nettement plus limitées de la temporalité des recherches subventionnées » (2015 : 155). En effet, la durée du statut pose la question de la notion de mission. Comment je gère ta présence et ce que tu prends en charge et apporte dans un temps limité ? Qui fera ensuite Parlemonde? Ta présence signifie pour la maison une mission. Or, cette mission est devenue un pilier du projet global de MA. Comment gérer le tempo autant que la temporalité dans cette dynamique?

31 Il s'agit donc de trouver comment dépasser le conflit des temporalités imbriquées par le contexte collaboratif de sa recherche: 1) le temps de la genèse d'un projet, 2) le temps de sa réalisation (les résidences), 3) le temps de sa valorisation (préparation des outils de communication, de presse, préparation des espaces d'exposition etc.), 4) le temps de l'exposition publique. Il faut à cela ajouter une cinquième étape installant un travail réflexif -critique, collaboratif et collectif, permettant de dépasser la remise en cause ou l'autoréflexivité au profit d'un «paradigme transactionnel » (Fabre 2014 : 75) invitant à passer à une action réfléchie. En parallèle, le doctorant doit recueillir et analyser son corpus, et rédiger son propre travail de thèse. En d'autres termes, il faut à la fois créer un espace de transformation où puissent émerger les discours du " pendant » et de "l'après », instaurant un ou plusieurs savoir-faire pour les projets à venir, et pouvoir s'en extraire pour avancer sa thèse.

Or, ces espaces-temps n'existaient pour aucun des partenaires, dont l'activité professionnelle cadence les calendriers, les plongeant d'une année à l'autre au cœur des autres projets déjà programmés, qu'il faut réaliser à temps. Et surtout, leur importance ou leur nécessité n'étaient pas conscientisées. Tout l'enjeu consiste donc à faire émerger des « dispositifs de co-explicitation » (Vinatier, 2010) : on fait d'autant mieux

Recherches en didactique des langues et des cultures, 17-2 | 2020 
son métier «qu'on le maitrise sur le plan conscient, qu'on est capable de porter à l'explication les principes pratiques qu'on met en œuvre dans sa pratique " (Bourdieu 2012 : 152). Pour porter ces éléments à la conscience des acteurs dans un contexte plurilingue et interculturel, une relation avec eux doit non seulement s'installer mais également être nourrie dans un continuum propice au développement d'échanges réciproques qui perdureront après la recherche.

\section{Conclusion : Parier sur la collaboration}

Du projet artistique à la recherche scientifique, il y a, plutôt qu'un hiatus ou un fossé, un écart fascinant, un espace médiant reliant diverses identités, langues et cultures professionnelles, dans un mouvement propre aux zones de créativité et d'interactions, cœurs des métiers des arts vivants. Collaborer c'est s'engager ensemble dans et vers un projet en construction, c'est donc accepter les incertitudes et les logiques de création qui ont pour caractéristique de ne pas répondre à des protocoles et qui n'ont pas de résultat connu par avance. C'est nourrir des élans communs, partager des valeurs, inventer un vocabulaire accessible à tous: c'est composer. Nous sommes passés collectivement du risque, moteur essentiel de créativité dans une structure culturelle et vecteur de biais pour le chercheur, au pari. Si «se former c'est se transformer " (Vinatier \& Morrissette, 2015 : 142), la particularité des recherches collaboratives nous semble résider dans la réciprocité de cette dynamique de transformation / trans-formaction et dans l'acceptation des dimensions subjectives et intimes de ce parcours. Cet article pourrait d'ailleurs en constituer une trace, à valeur performative, explorant à deux voix l'idée d'un continuum et d'un déplacement qui, pour être fécond, repose sur les expériences (conscientisées et explicitées) et les histoires professionnelles et personnelles de tous les acteurs. La posture de « chercheur embarqué » met en lumière des points d'invisibilité interculturels et langagiers pouvant générer des zones de résistances ou des interférences, mais ces dernières ne sont pas indépassables, ni incompatibles avec la recherche. Au contraire, elles la nourrissent.

Jongler avec les nécessités d'une pratique opérationnelle et les exigences d'un travail de thèse ancré dans le paradigme de la «low science » et de l'épistémologie de la relation, est corrélé à une autre forme d'exigence partagée, qui n'a plus rien à voir avec la livraison d'une méthodologie clé en mains ou d'un protocole efficient ni pour l'entreprise, ses partenaires ou pour le doctorant. Tous co-embarqués, nous ne fabriquons pas de produit mais des expériences artistiques, uniques, vivantes, mouvantes, qui, bien qu'institutionnellement et professionnellement encadrées, se nourrissent à la fois du professionnalisme et de la sensibilité de chacun. Le travail déployé dans ces lieux de création s'émancipe donc d'une logique proprement économique et procède de ce que Menger définit comme : "la réalisation de soi dans l'agir productif » (2019). Il s'agit pour la doctorante de faire émerger les conditions de cette conception expressive du travail chez tous les acteurs, en interrogeant ce qui circule consciemment ou non, dans quelles configurations, et d'analyser la place des langues (familiales, professionnelles, etc.) dans cette dynamique de " compagnonnage " (Narcy-Combes, 2001). C'est à cet endroit que se rejoignent les travaux du «chercheur qualitatif », de l'artiste, de l'enseignant et du participant, engagés ensemble par la création, au service de valeurs communes rendues explicites par l'effort de réflexivité éthique (Dewey, 1934) conditionnant un agir ensemble public. Les échanges, les 
« interactions » sont devenus des activités expressives complexes et sensibles, livrés de façon formelle ou non. Ils sont la condition d'émergence de toute création, artistique comme scientifique, à condition d'en accepter, voire d'en revendiquer, la part d'imprévisible, d'intersubjectif et de mouvant.

\section{BIBLIOGRAPHIE}

Alam, T., Gurruchaga M., \& O’Miel, J. (2012). « Science de la science de l'État : la perturbation du chercheur embarqué comme impensé épistémologique. » Sociétés contemporaines, 87, 155-173.

Agier, M. (2004). La sagesse de l'ethnologue. Paris, L'œil neuf.

Auger, N. (2001). «Intersubjectivité et interculturalité. Une enquête sociolinguistique sur l'apprentissage du français par le théâtre ». LACIS Subjectivités, Singularités, Cultures, n³, 95-117.

Auger, N., Terrades, O.\& Talagrand, R. (2007). « Ateliers littérature et arts pour entrer dans le français langue seconde. » ELA. Études de linguistique appliquée, 147(3), 339-348.

Auger, N. (2007). Construction de l'interculturel dans les manuels de langues. Fernelmont, Éditions Modulaires Européennes.

Auger, N., Dervin, F. \& Suomela-Salmi, E. (2009). Pour une didactique des imaginaires dans l'enseignement-apprentissage des langues étrangères. Paris, L'Harmattan.

Bamford, A. (2008). « Mesurer l'impact : Recherche(s) en éducation artistique et culturelle ». Dans Ministère de l'Éducation nationale et Ministère de la Culture (dir.), Évaluer les effets de l'éducation artistique et culturelle. Paris, La Documentation française, Centre Pompidou.

Bednarz, N. (2015). « La recherche collaborative. » Carrefours de l'éducation, n³9, 171-184.

Blanchet, P. (2012). La linguistique de terrain : méthode et théorie. Rennes, Presses universitaires de Rennes.

Blondiaux, L., Fourniau, J-M. \& Mabi, C. (2016). « Chercheurs et acteurs de la participation : liaisons dangereuses ou collaborations fécondes? » Participations, n¹6(3), 5-17.

Bourdieu, P. (2012). «Sur l'État», Cours au Collège de France, 1998-1992. Paris, Seuil.

Bordeaux, M-.C. (2017). « L'éducation artistique et culturelle à l'épreuve de ses modèles. » Quaderni, 92, 27-35.

Bordeaux, M-C. (2014). «Les aléas de l'éducation artistique et culturelle : brèves rencontres, rendez-vous manqués et avancées territoriales [en ligne] ». Comité d'histoire du ministère de la Culture et de la Communication, Centre d'histoire de Sciences-Po Paris, La démocratisation culturelle au fil de l'histoire contemporaine. Paris, 2012-2014.

De Lavergne, C. (2007). « La posture du praticien-chercheur : un analyseur de l'évolution de la recherche qualitative ». Recherches qualitatives, Hors Série 3, 24-43.

Dewey, J. (2010/1934). L'art comme expérience. Paris, Gallimard. 
Dupont, N. (2010) « Les partenariats écoles / institutions culturelles : des passages entre cultures juvéniles, cultures artistiques et cultures scolaires? » Les Sciences de l'éducation-Pour l'Ère nouvelle, $43,95-121$.

Fabre, M. (2014). « Minimalisme moral et maximalisme éthique chez John Dewey. » Recherches en Éducation, Hors série $\mathrm{N}^{\circ} 6,71-80$.

Ferrando u Puig , J. \& Petit, G. (2016). « Les usages de la réflexivité dans l'entreprise participative. Un enjeu identitaire aux implications pratiques, théoriques et marchandes. » Participations, $\mathrm{n}^{\circ} 16$, 19-43.

Hellec, F. (2014). « Le rapport au terrain dans une thèse CIFRE. » Sociologies pratiques, 28(1), 101-109.

Kerlan, A. \& Lemonchois, M. (2017). « Les projets artistiques, vecteur de formation des enseignants? » Recherche \& formation, 86(3), 9-12. Disponible à : https://www.cairn.info/revuerecherche-et-formation-2017-3-page-9.htm.

Menger, P-M. (2019). « Comment achever une œuvre ? Travail et processus de création » Introduction, Cours au Collège de France. Disponible à : https://www.college-de-france.fr/site/ pierre-michel-menger/course-2018-2019.htm.

Narcy-Combes, J-P. (2001). « Recherche et pratiques pédagogiques en langues de spécialité. » Cahiers de l'APLIUT, Vol.21, $\mathrm{n}^{\circ} 2$.

Pierra, G. (2006). Le corps, la voix, le texte, arts du langage en langue étrangère. Paris, L'Harmattan. Pourtois, J.-P. \& Desmet, H. (2009). « Objectivité en sciences humaines » dans Mucchielli A. (éd.) Dictionnaire des méthodes qualitatives en sciences humaines et sociales. Paris, Armand Colin.

Sérusclat-Natale, M. \& Adam-Maillet, M. (2018). « Le projet artistique : une puissance maïeutique de la compétence langagière. » LIDIL $\mathrm{N}^{\circ} 57$ Démarches créatives, détours artistiques et appropriation des langues. Disponible à : https://journals.openedition.org/lidil/4908.

Vinatier, I. \& Morrissette, J. (2015). « Les recherches collaboratives : enjeux et perspectives. » Carrefours de l'éducation, n³9, 137-170.

Vinatier, I. (2010). « L'entretien de co-explicitation entre chercheurs et enseignants : une voie d'émergence et d'expression du sujet capable. » Recherches en éducation, $\mathrm{n}^{\circ} 1,111-129$.

\section{NOTES}

1. Site compagnon du projet : http://parlemonde.mascenenationale-creative.com.

2. Project partner site : http://parlemonde.mascenenationale-creative.com.

3. Lorsque nous avons écrit cet article, souvent à distance, notre co-écriture a pris la forme d'un dialogue plus personnel, dont on trouve les traces syntaxiques (pronoms, marques d'adresse, questions en suspens, etc.) dans les extraits qui vont suivre. C'est volontairement que nous laissons apparaître ce glissement sémantique puisqu'il est une manifestation concrète de la pensée que nous développons dans cette contribution.

4. On trouvera ici le site compagnon de Parlemonde avec des traces des 2 éditions de ce festival : Parlemonde 1 en 2017 et le programme de Parlemonde 2 qui a eu lieu en mai 2019 : http:// parlemonde.mascenenationale-creative.com.

5. https://www.scenes-nationales.fr/label/.

6. https://www.education.gouv.fr/pid25535/bulletin_officiel.html ?cid_bo =71673. 
7. MA signifie ici Montbéliard Agglomération et vient souligner l'importance accordée par la direction à l'appropriation de cette institution culturelle par les habitants du territoire.

8. Une CIFRE est un contrat de travail qui lie le doctorant, l'entreprise qui le salarie, le laboratoire de recherche et le ministère de la recherche via l'ANRT (association nationale recherche technologie). L'entreprise reçoit une subvention qui prend en charge une partie du salaire du doctorant.

9. Cf. Loi $n^{\circ}$ 2013-595 du 8 juillet 2013 d'orientation et de programmation pour la refondation de l'école de la République et la circulaire $n^{\circ}$ 2012-141 du 2-10-2012.

10. Voir par exemple: http://www.culture.gouv.fr/Thematiques/Langue-francaise-et-languesde-France/Politiques-de-la-langue/Maitrise-de-la-langue/Action-culturelle-et-maitrise-dufrancais.

11. Formulation choisie par le directeur de la scène nationale, réemployant ici le qualificatif que l'on donne aux artistes qui vont collaborer de manière étroite pendant une ou plusieurs saisons (" artistes associés »), et que la structure met en valeur.

12. C'est à ce moment que notre co-écriture a pris la forme d'un dialogue plus personnel comme le montrent les marques d'adresse. Cela témoigne de l'importance de «l'effet relationnel » dans une recherche collaborative.

13. D pour doctorante, ENT pour entretien, A1 pour artiste 1 et A2 pour artiste2.

14. Phrase prononcée par ESIA12, élève UPE2A de lycée professionnel, participant à Parlemonde 2.

15. Dans la continuité de cette logique relationnelle et subjective, j'emploierai dans les trois prochains paragraphes volontairement le «je» pour retracer ce qui, dans mon parcours personnel, a nourri et suscité mon actuelle situation de doctorante associée à MA.

\section{RÉSUMÉS}

Cette contribution s'appuie sur une recherche en sciences du langage en cours, menée dans le cadre d'un doctorat sous contrat CIFRE (Convention Industrielle de Formation par la REcherche) au sein d'une scène nationale, lieu de création et de diffusion artistique. En 2016, une collaboration s'installe entre la scène nationale du pays de Montbéliard et l'éducation nationale et prend la forme d'un festival de créations participatives: Parlemonde ${ }^{1}$. A travers une expérience commune de création, se rencontrent des élèves allophones nouvellement arrivés, des enseignants, des artistes européens pluridisciplinaires, une chercheuse et des spectateurs. Cette contribution s'attachera à montrer comment cette collaboration particulière a considérablement déplacé les participants : le projet est devenu un vaste espace de jeux, au sens de collaboration dynamique. Les questionnements, les attentes, les pratiques et les positions de chaque interlocuteur (doctorant compris) sont sans cesse en mouvement.

Nous examinerons la place du chercheur dans cette collaboration, dont l'activité conventionnée est soumise à des règles. Quelles règles du jeu co-construire avec les équipes de la structure qui salarie le doctorant pour installer un (ou plusieurs) savoir-faire, qu'aucune des parties impliquées ne pourrait, seule, mettre en jeu? On s'interrogera ensuite sur les espaces de friction ou de jeu au sens d'intervalle "entre deux pièces d'un même mécanisme " en s'intéressant particulièrement à ce que recouvre la collaboration pour le chercheur et ses données dans un tel contexte. Afin de laisser entrevoir au lecteur quels enjeux sont à l'œuvre dans ces contextes mouvant et agissant au cœur de l'espace social, on abordera ces questions en laissant deux voix 
dialoguer: celle du directeur de la scène nationale, et celle de la doctorante salariée par la structure.

This paper is based on a research situation in progress in the Language Sciences that was carried out in a national theater, a place for artistic creation and diffusion, as part of a Doctoral thesis under the CIFRE (Convention of Industrial Training through Research) agreement. A collaboration was initiated in 2016 between the Ministry of National Education and a common artistic project, Parlemonde ${ }^{2}$. Meetings were organized between minority-language speaking students newly arrived in France, their teachers, multidisciplinary European artists, researcher and spectators in a joint creative experience. This collaboration is special because it is the first of its kind, requiring the expertise of each of the stake holders of the project (artists, teachers and students). All of them were moved to a vast games area involving a collaborative dynamics where questions, expectations, practices and positions of each of the players (including this researcher) were constantly in motion. The researcher's place in this collaboration will be examined through a study of the "game" as a conventional activity subject to collaborative rules between the participants. What then are the rules to be co-constructed with the teams belonging to the organization funding the researcher and whose objectives are to develop a (or several) skills that none of the partners are in a position to develop by themselves? The consequences for the researcher and the organization will be examined by considering the game as an interval between two parts of the same process, an ideal interstitial space facilitating mediation, focusing particularly on the development of the researcher's "game" in this context. The paper will examine these questions through a dialogue of two voices: that of the Director of the regional theater, and of the researcher funded by the organization, in order to allow the reader to understand the stakes that are "at play" in this type of fluid and changing context found at the heart of the social sphere.

\section{INDEX}

Mots-clés : jeu, médiation, formation, conscientisation, plurilinguisme

Keywords : game, mediation, training, awareness, plurilingualism

\section{AUTEURS}

\section{MAUD SERUSCLAT-NATALE}

LHUMAIN, Université Paul Valéry Montpellier III \& CIFRE à MA scène nationale du Pays de Montbéliard

M. Sérusclat-Natale est certifiée de lettres, FLE/FLES et théâtre, a collaboré avec le CASNAV et la DAAC de l'académie de Besançon. Elle travaille actuellement à MA, scène nationale de Montbéliard sur Parlemonde, projet artistique plurilingue auquel elle consacre un doctorat en sciences du langage, dirigé par N. Auger (Université Paul Valéry, Montpellier).

m.natale[at]mascene.eu

\section{YANNICK MARZIN}

Directeur MA scène nationale - Pays de Montbéliard

Y. Marzin dirige la scène nationale du Pays de Montbéliard en 2011 après des postes de direction au Ballet du Nord avec Carolyn Carlson, et au Conservatoire National Supérieur de Musique et de Danse de Paris. Formé à la danse, il est titulaire d'une maîtrise en géographie, d'un D.E.A. en esthétique et d'un D.E.S.S en politiques culturelles. Il a enseigné à Paris 10 pendant 10 ans et été 
consultant pour divers festivals en Europe.

ym[at]mascene.eu 